\title{
Development of motor imagery ability in children with developmental coordination disorder - A goal-directed pointing task
}

\author{
Imke L. J. Adams '* D, Jessica M. Lust' and Bert Steenbergen ',2,3 \\ 'Behavioural Science Institute, Radboud University, Nijmegen, The Netherlands \\ ${ }^{2}$ School of Psychology, Australian Catholic University, Sydney, New South Wales, \\ Australia \\ ${ }^{3}$ Centre for Disability and Development Research, Australian Catholic University, \\ Sydney, New South Wales, Australia
}

\begin{abstract}
Children with developmental coordination disorder (DCD) have difficulties with the predictive control of movements. This was shown in studies that target motor imagery and motor planning, and appears to become particularly evident with increases in task complexity. In this study, we used a complex mental chronometry paradigm to examine the development of motor imagery ability in children with DCD, using a longitudinal design. Thirty children were included in the DCD group (aged 6-II years) and age- and gender-matched to 30 controls. The DCD group had a Movement Assessment Battery for Children-2 score $\leq 16$ th percentile and the control group $\geq 25$ th percentile. Results of this study showed that children with DCD indeed had a significantly lower correlation between executed and imagined movements. Importantly, the increase in the correlation and linear fit during subsequent measurements was comparable for the DCD and control group. Together, these findings suggest a delayed developmental onset of motor imagery ability in DCD, but a similar rate of development over time compared to the control group. Based on these results, it seems likely that explicit motor imagery instructions can be used to improve predictive control in children with DCD.
\end{abstract}

Children with developmental coordination disorder (DCD) have difficulties performing coordinated movements (American Psychiatric Association, 2013). Consistent with the internal modelling deficit hypothesis (Wilson \& Butson, 2007), earlier studies have indicated that this is due to problems with the predictive control of movements (Adams, Lust, Wilson, \& Steenbergen, 2014; Wilson, Ruddock, Smits-Engelsman, Polatajko, \& Blank, 2013). Predictive control of movements is guided by the use of sensory predictions of the outcome of an intended movement in a feedforward manner. An internal model of a movement builds such a sensory prediction (Wolpert, 1997). Recent studies show that the deficit in predictive control in children with DCD is more task specific than was originally thought (Wilson et al., 2017). Variation in the pattern of performance (relative to controls) is shown according to the complexity of the task in case of motor imagery and planning (Adams, Ferguson, Lust, Steenbergen, \& Smits-Engelsman, 2016; Fuelscher, Williams, Wilmut, Enticott, \& Hyde, 2016; Noten, Wilson, Ruddock, \& Steenbergen,

*Correspondence should be addressed to Imke L. J. Adams, Radboud University, Spinozagebouw A (A.04.23A), PO Box 9104, 6500 HE Nijmegen, The Netherlands (email: i.adams@pwo.ru.nl). 
2014). Specifically, studies on motor imagery in DCD have employed two paradigms: implicit motor imagery using a mental rotation paradigm (e.g., Adams, Lust, Wilson, \& Steenbergen, 2017b; Deconinck, Spitaels, Fias, \& Lenoir, 2009; Williams, Omizzolo, Galea, \& Vance, 2013; Williams, Thomas, Maruff, \& Wilson, 2008) and explicit motor imagery where a mental chronometry paradigm is employed (e.g., Ferguson, Wilson, \& SmitsEngelsman, 2015; Lewis, Vance, Maruff, Wilson, \& Cairney, 2008; Williams et al., 2013). Collectively, these studies suggest that motor imagery is compromised in children with DCD, but the extent to which this is the case varied across the different paradigms. Different outcomes are likely due to different task instructions (e.g., whether direct instructions to use motor imagery are given in the hand rotation task), stimuli (hand rotation vs. whole-body rotation tasks) and task presentation (e.g., mental chronometry on a tablet vs. on paper). Hétu et al. (2013) have shown that mental rotation paradigms and mental chronometry paradigms tap distinct forms of motor imagery and rely on different neural networks. In this study, the development over time of explicit motor imagery is examined in children with DCD.

In explicit motor imagery tasks, participants are asked to imagine and feel themselves making movements from a first-person, egocentric perspective (Decety \& Grezes, 1999; Gabbard, 2009). In the mental chronometry paradigm, chronometry between mentally imagined and physically executed actions is taken as evidence for the use of motor imagery (Decety, Jeannerod, \& Prablanc, 1989). Most often arm or hand movements are used for the mental chronometry task, but use of motor imagery has also been shown in mental chronometry tasks examining walking (e.g., Spruijt et al., 2013). The mental chronometry paradigm requires a more explicit form of imagery, with visual guidance and involving speed-accuracy components (Williams et al., 2013), than, for example, the hand rotation task. Prominent examples include tasks such as the imagined pointing tasks, which include the visual guided pointing task (VGPT; Maruff, Wilson, Trebilcock, \& Currie, 1999; Sirigu et al., 1996) and the computerized visual radial Fitts' task (C-VRFT; Caeyenberghs, Tsoupas, Wilson, \& Smits-Engelsman, 2009; Caeyenberghs, Wilson, van Roon, Swinnen, \& Smits-Engelsman, 2009; Ferguson et al., 2015; Smits-Engelsman \& Wilson, 2013). Earlier research, using the VGPT, showed that in typically developing children the movement durations of imagined pointing movements obey to Fitts' law (Fitts, 1954), in a similar manner as executed movements. In contrast, in children with DCD only durations of actual movements obeyed to Fitts' law and not imagined movements (Lewis et al., 2008; Maruff et al., 1999; Williams et al., 2013; Wilson, Maruff, Ives, \& Currie, 2001). More recently, Ferguson et al. (2015) compared movement durations of typically developing children and children with DCD on two tasks: the VGPT and the C-VRFT. The C-VRFT requires a sequence of five back-and-forth movements to distinct targets located on a radial axis. The movement trajectory is more varied and thereby imposes a higher motor planning demand than the back-and-forth movement to one single target in the VGPT (Ferguson et al., 2015). The correlation between executed and imagined movement durations was significantly lower for the DCD group than the control group, on both tasks (Ferguson et al., 2015). However, group differences were more pronounced in the imagined condition of the radial Fitts' task (C-VRFT) compared to the traditional VGPT. Poor predictive control (viz. motor planning) in children with DCD thus appears to be more evident for tasks that impose a higher demand on motor planning (Adams et al., 2017b; Fuelscher et al., 2016; Noten et al., 2014; Wilson et al., 2017).

In implicit motor imagery tasks, participants are not specifically instructed to use motor imagery to solve the task. The hand rotation task has often been used to examine implicit motor imagery ability (e.g., Deconinck et al., 2009; ter Horst, van Lier, \& 
Steenbergen, 2010). During this task, participants are asked to determine whether presented stimuli are a right or a left hand. When response times and number of errors are in line with the biomechanical constraints experienced during real movements (increased response time and number of errors when laterally orientated are presented), use of motor imagery is inferred. When comparing implicit motor imagery (hand rotation task) performance of young adults with probable DCD (pDCD; Hyde et al., 2014) to younger children with pDCD (e.g., Fuelscher, Williams, Enticott, \& Hyde, 2015), it appears that children with DCD are able to improve their implicit motor imagery ability as they age. Data on the development of explicit motor imagery performance are currently lacking. Longitudinal data about motor imagery ability in children with DCD are crucial to design targeted motor imagery programmes (Adams, Steenbergen, Lust, \& Smits-Engelsman, 2016; Wilson, Thomas, \& Maruff, 2002; Wilson et al., 2016). A recent study on the development of implicit motor imagery ability (using the hand rotation task) showed that children with DCD were slower and less accurate than controls in all subsequent years but were able to improve their motor imagery ability over time at the same rate as controls (Adams, Lust, Wilson, \& Steenbergen, 2017a).

In this study, we used the C-VRFT to be able to elicit group differences and follow development of explicit motor imagery ability over time. Our longitudinal set-up to study the development of explicit motor imagery in DCD will inform the discussion as to whether the deficit in predictive control observed in DCD reflects immaturity of the motor system or some deviation from normal development (Hyde \& Wilson, 2013; Ruddock et al., 2015). A developmental delay of explicit motor imagery in children with DCD is inferred when the DCD group shows impaired motor imagery abilities compared to their typically developing peers, but develops these skills at the same rate (or faster, in case of a catch-up) as their peers (Hyde \& Wilson, 2013). A disorder (deviance from the normal developmental continuum) is inferred when the developmental trajectory of the children with DCD significantly differs from their typically developing peers (Hyde \& Wilson, 2013). Annaz, Karmiloff-Smith, and Thomas (2008) provided more in-depth descriptors of a developmental delay, by looking at developmental trajectories. A delayed onset implies a normal rate of development but a delayed start of the development compared to controls. A delayed rate implies a normal onset but slower rate of increase in performance, while a delayed rate and onset implies both a delayed start of the development and a slower rate of increase in performance (Annaz et al., 2008; Thomas et al., 2009). Earlier literature about the developmental trajectories in DCD referred to delay versus disorder (Hyde \& Wilson, 2013; Ruddock et al., 2015), combining this with the more elaborated description of Annaz et al. (2008), it seems that what is called a disorder in fact reflects a zero rate or slower rate of increase in performance. Results in the recent study on the development of implicit motor imagery ability, using the hand rotation task, indicated a delayed onset of this ability (Adams et al., 2017a).

In clinical practice, a mixture of implicit and explicit instructions are used, but especially during the early learning phase of movement the main focus is usually on explicit instructions (Steenbergen, van der Kamp, Verneau, Jongbloed-Pereboom, \& Masters, 2010). More insight into the development of explicit motor imagery in children with DCD will therefore inform these intervention programmes.

This study aimed to examine the development of explicit motor imagery skills over time in children with DCD compared to age-matched controls using the C-VRFT. We tested 30 children with DCD (according to DSM-V criteria) and 30 gender- and agematched controls. To be able to include enough children with DCD that conform to the 
clinical DSM-V criteria for DCD, we included children aged 6-11 years. Children participated in three measurements (T0, T1, and T2) with approximately 1 year in between measurements. Motor ability, using the Movement Assessment Battery for Children (mABC-2, Henderson, Sugden, \& Barnett, 2007; - Dutch translation, SmitsEngelsman, 2010), and explicit motor imagery using the C-VRFT were assessed at all three time points. The mABC-2 includes measures of fine motor ability, gross motor ability and balance. We hypothesized that the DCD group would have a lower correlation between executed and imagined movements during the first measurement (T0), compared to controls. Based on recent cross-sectional studies that made a comparison between age groups (Fuelscher et al., 2015; Hyde et al., 2014) and a recent longitudinal study on implicit motor imagery in DCD (Adams et al., 2017a), we hypothesized that children with DCD show a delayed onset but similar rate of development of explicit motor imagery over time as the control group.

\section{Methods}

\section{Participants}

Thirty children (23 boys) between the ages of 6 and 11 years (T0) who met the DSM-V diagnostic criteria for DCD were included in this study, and gender- and age-matched ( \pm 4 months) to controls. Mean age for the DCD group was 8.87 years $(S D=1.40)$ and 8.85 years $(S D=1.40)$ for the control group at T0. Handedness was assessed by performing the manual tasks of the $\mathrm{mABC}-2$ and also indicated by their parents on the health questionnaire. Two children in the DCD group and four children in the control group were left-handed, and all other children were right-handed.

The DCD group was recruited through paediatric physical therapists and via Balans (Dutch organization for parents of children with DCD) through an advertisement on their website. In the first year, 33 children with DCD participated, gender- and age-matched to 33 controls (this population is also described in Adams et al., 2017a, 2017b). In subsequent years, 30 children with DCD were able to participate at all three time points, and gender- and age-matched to 30 controls. The DCD children all met the following four inclusion criteria to ensure that the DSM-V diagnostic criteria were met: (1) MABC-2 (Dutch translation - Smits-Engelsman, 2010) total percentile score $\leq 16$ th (criterion A DSM-V), (2) treated or have been treated for a motor coordination problem by a paediatric physical therapist. In addition, parents filled out the DCDQ (Dutch translation; Schoemaker, Reinders-Messelink, \& de Kloet, 2008) to monitor the interference of the motor problem with activities of daily living and academic productivity (criterion $B$ of DSM-V), (3) IQ > 70. If children attended regular primary education and had not been diagnosed with a learning disorder, an IQ $>70$ was inferred. When children attended special education, IQ score was checked by asking their parents the latest IQ score (criterion D DSM-V), and (4) no visual impairments or neurological conditions that could affect their motor abilities (criterion D DSM-V). A health questionnaire with specific questions about medical conditions was used to ascertain this last criterion. Criterion $C$ of the DSM-V about the early onset of symptoms is present because the children have symptoms between 6 and 11 years of age. Three children in the DCD group had a diagnosis ADHD. As attentional problems might influence the performance of the experimental tasks (Fong et al., 2016), three children in the DCD group with an ADHD diagnosis were excluded, as well as their gender-and age-matched controls. This yielded a total of 27 children in the DCD group and 27 children in the control group. The mABC-2 
percentile scores of the children with DCD ranged from 0.1 to 16.0 (Total Test Score $M=50.63, S D=14.22$ ).

The control group was recruited on two mainstream primary schools. Control children were included if they had a mABC- 2 total percentile score $\geq 25$ th and IQ $>70$. An IQ $>70$ was inferred when attending regular primary education and no presence of a learning disorder. The mABC-2 percentile scores were in the range of 25-98 (Total Test Score $M=81.41, S D=7.28$ ).

\section{Experimental task - C-VRFT}

Participants were seated on a comfortable chair with their arms resting in front of them. Custom-developed software in the Presentation software package (Neurobehavioral Systems, Albany, CA, USA) was used to present stimuli and record data. A 19-inch touch screen (ELO 1928L Desktop Touchmonitor, Elo TouchSystems, Menlo Park, CA, USA) was placed in front of participants on a table and was tilted about $10^{\circ}$ towards them. The CVRFT requires a sequence of five back-and-forth movements to distinct target located on a radial axis from a home base. In the C-VRFT, five target circles were displayed on a touch screen on 75- or 150-mm-long radials from a central home base between a green start box and red stop box (Figure 1; Caeyenberghs, Wilson, et al., 2009). Varying target distances $(A)$ - the distance between the middle of the home base and the edge of the five radials and target widths $(W)$ were used, resulting in four different task difficulties (i.e., index of difficulty (ID), Table 1$)$, as computed by ID $=\log 2(2 \times(A / W))$ (Fitts, 1954). Participants were requested to move (execution condition) or to imagine moving (imagery condition) in straight lines between the central home base and each of the five target circles. In both the execution and imagery conditions, participants had to touch the start button first. Subsequently, a beep sound was presented (after a random duration between 500 and 1,000 ms). For the execution condition, participants were required to move back

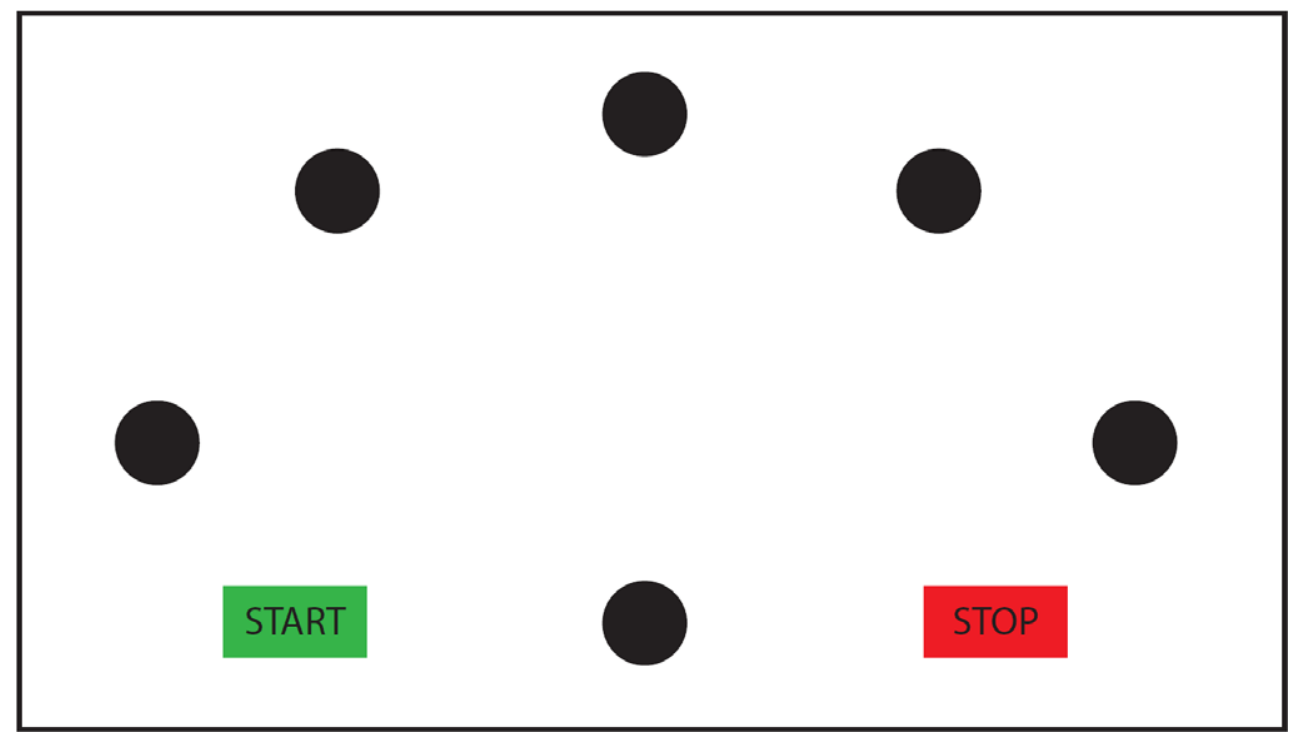

Figure I. Schematic representation of the computerized visual radial Fitts' task. [Colour figure can be viewed at wileyonlinelibrary.com] 
Table I. Indices of difficulty in the computerized visual radial Fitts' task

\begin{tabular}{lcc}
\hline Index of difficulty & Target distance $(\mathrm{mm})$ & Target size $(\mathrm{mm})$ \\
\hline 3.32 & 75 & 30 \\
4.32 & 150 & 30 \\
5.32 & 75 & 7.5 \\
6.32 & 150 & 7.5 \\
\hline
\end{tabular}

and forth between the consecutive radial targets and the central home base in fixed order from left to right and finally move with their index finger to the stop button (Figure 1). They were instructed to do so as fast and as accurately as possible. Participants had to perform the task using the index finger of their dominant hand. We decided to use the index finger to control the touch screen instead of a (electronic) pen because handwriting problems are well known in children with DCD (Prunty, Barnett, Wilmut, \& Plumb, 2013; Rosenblum \& Livneh-Zirinski, 2008). Finger movements were recorded at a frequency of $100 \mathrm{~Hz}$. For the imagery condition, the participants were required to imagine their movements back and forth to the same five targets, while their index finger remained at the central home base. When they finished imagining touching all five targets, they had to move their index finger to the stop button. Each child received a demonstration by the experimenter and two practice trials at the beginning of both blocks. The children first performed the execution condition, followed by the imagery condition. Previous work in adults has shown that condition order does not affect performance (Papaxanthis, Pozzo, Skoura, \& Schieppati, 2002). There were three repetitions for each ID, resulting in 12 randomly ordered trials in both conditions.

\section{Procedure}

Approval for the experiment was obtained from the local Ethical Committee (Registration number: 2013-1405-110a1). The parents of all participants signed a written informed consent prior to the study and were asked to fill in the DCDQ (Dutch translation Schoemaker et al., 2008), the ADHD questionnaire (Scholte \& van der Ploeg, 2004), and a questionnaire concerning the health of their child. The ADHDQ was used to examine signs of ADHD (attention, hyperactivity, impulsivity) in both groups, because ADHD often co-occurs in DCD (Goulardins et al., 2015). After receiving the informed consent and questionnaires, the child was asked to fulfil the C-VRFT and the mABC-2 (SmitsEngelsman, 2010). The C-VRFT and MABC-2 were repeated at measurement T1 and T2.

\section{Data analysis}

All analyses were performed using SPSS version 21 (IBM Corporation, Armonk, NY, USA). Alpha level was set at .05, and Greenhouse-Geisser corrected values were reported when the assumption of sphericity was violated. Total scores on the DCDQ and ADHDQ were compared between the DCD and control group with two Mann-Whitney $U$-tests. Mean movement duration (time between touching the start button and reaching the stop button) was calculated for each ID and each child separately and for the execution and imagery condition separately. The analyses were performed in three steps: (1) Temporal congruence between execution and imagery conditions was determined by Pearson correlation for the movement duration of the two conditions, both at group level and for 
each individual. Correlations were then Fisher-Z transformed and subjected to a repeatedmeasures ANOVAs with time (T0, T1, T2) as repeated factor and group as between-subject factor, to test whether the temporal congruence was different between the DCD and control group over time; (2) The effect of ID on movement duration in both conditions and in both groups on the three measurement points (T0, T1, T2) was examined with a repeated-measures ANOVA with ID (2.32, 3.32, 4.32, \& 5.32), condition (execution and imagery), and time (T0, T1, T2) as repeated factors and group as between-subject factor. (3) Compliance with Fitts' law (Fitts, 1954) was analysed using the goodness of fit $\left(R^{2}\right)$ and slope of the linear regression for the movement duration and ID, for each participant separately. Two repeated-measures ANOVA on goodness of fit and slope were used to test whether the linearity of the movement duration over the different indexes of difficulty differed between group (DCD/control) and condition (execution/imagery). Furthermore, one sample $t$-tests were used to test whether the $R^{2}$ for the actual and imagery movement performance were larger than 0 for children in each group separately.

\section{Results}

\section{Questionnaires}

Total scores on the DCDQ at baseline were lower for the DCD group (median $=38.0$ ) than the control group (median $=67.0), U=25.50, p<.001, r=-.80$. Total scores on the ADHDQ at baseline were higher for the DCD group (median $=22.0$ ) than for the control group $($ median $=9.0), U=161.0, p<.001, r=-.48$.

\section{Experimental task - C-VRFT}

For the C-VRFT, Pearson correlations between the duration of executed and imagined movements are displayed in Figure 2 . Because the Pearson correlations were not normally distributed, the Fisher-Z-transformed correlations were used for the repeated-measures ANOVA with group (DCD/control) as the between-subjects variable and time (T0, T1, T2) as the within-subjects variable. The DCD group had a significantly lower correlation than the control group, $F(1,52)=4.709, p=.035, \eta^{2}=.083$. The correlation between executed an imagined movements increased with time in both groups, $F(2,104)=4.106$, $p=.019, \eta^{2}=.073$. There was no difference between groups in the increase in the correlation over time, $p=.719$. Pearson correlations are displayed in Figure 2 .

The effect of index of difficulty (ID) on movement time in both conditions and in both groups was examined with a repeated-measures ANOVA with group as between-subject factor and time (T0, T1, T2), condition (execution and imagery), and ID (2.32, 3.32, 4.32, $\& 5.32$ ) as repeated factors. Because movement times in both conditions, for both groups, were not normally distributed, a $\log 10$ transformation was conducted on all movement times before running the analyses. There was no main effect of group, $F(1,52)=0.525$, $p=.47, \eta^{2}=.010$. Movement time decreased over time, $F(2,104)=4.48, p=.014$, $\eta^{2}=.079$, and this did not differ between groups, interaction time*group, $F(2,52$, $p=.47, \eta^{2}=.014$. Executed movements were performed more slowly than the imagined movements, $F(1,52)=99.56, p<.001, \eta^{2}=.657$. There was a trend that this difference in movement time between executed and imagined movements was larger in the DCD group than in the control group, $F(1,52)=3.519, p=.066, \eta^{2}=.063$ (see also Figure 3 ). Movement time significantly increased with increasing ID, $F(2.23,115.89)=227.43$, $p<.001, \eta^{2}=.814$. The time* condition interaction was significant, $F(1.80$, 


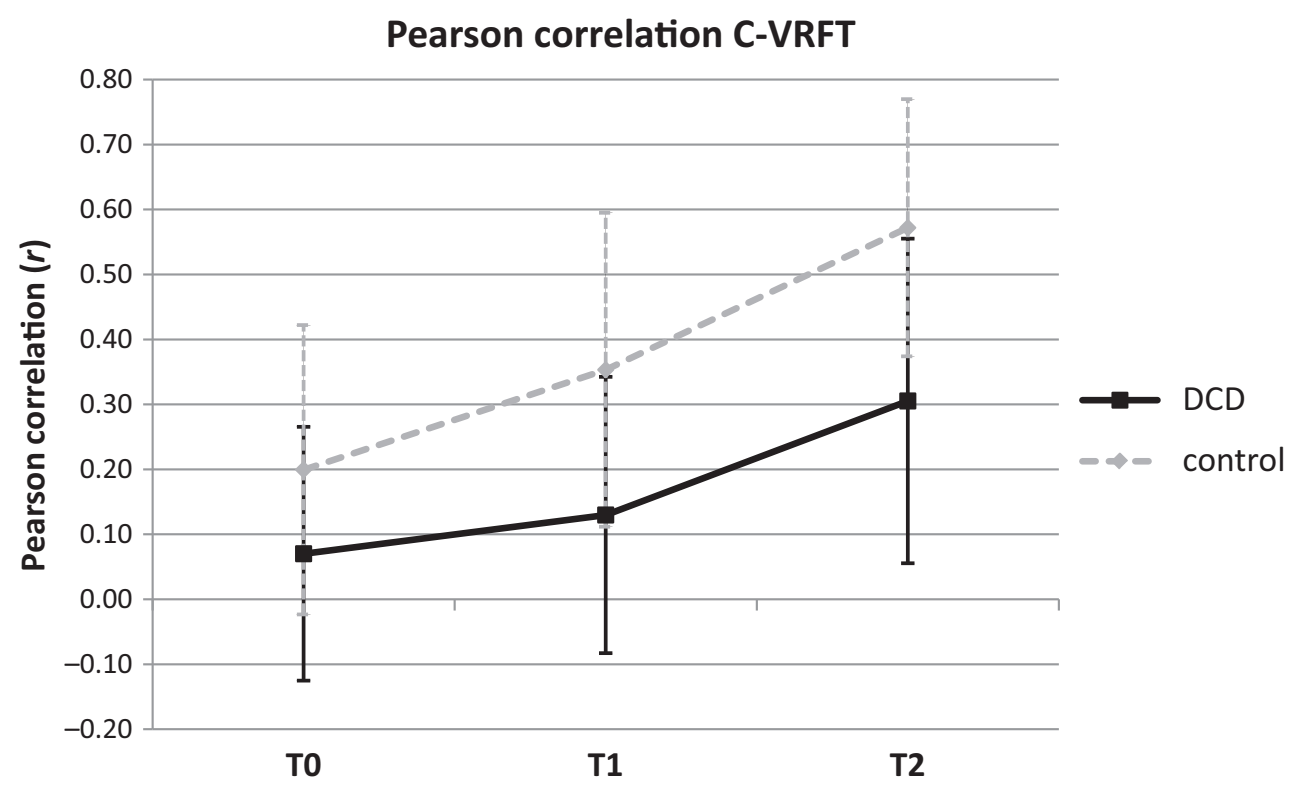

Figure 2. Pearson correlation computerized visual radial Fitts' task (C-VRFT) between executed and imagined movements at all three time points. Solid line (black) refers to developmental coordination disorder (DCD) group, and dashed line (grey) refers to control group.

$93.57)=36.54, p<.001, \eta^{2}=.413$, and separate analyses for executed and imagined movements showed that the movement time of executed movements decreased over time, $F(2,104)=22.80, p<.001, \eta^{2}=.305$, while the movement time of imagined movements increased over time, $F(2,104)=8.58, p<.001, \eta^{2}=.412$ (see Figure 3). The interaction condition $*$ ID was significant, $F(2.25,116.83)=124.00, p<.001$, $\eta^{2}=.706$. Separate analyses for executed and imagined movements showed that movement time of executed movements increased with increasing ID, $F(3$, $156)=353.76, p<.001, \eta^{2}=.872$. For imagined movements, movement time also increased with increasing ID, but this effect was less strong, $F(3,156)=21.59, p<.001$, $\eta^{2}=.293$. No other interactions were significant at an alpha level of .05. The increase in movement time per ID for executed and imagined movements is displayed in Figure $3 \mathrm{~A}$ (DCD group) and 3B (control group).

Goodness of fit deviated significantly from 0 for participants in both the DCD and control group at all three measurement occasions (see Table 2). Goodness of fit was examined with a repeated-measures ANOVA with time (T0, T1, T2) as the within-subjects variable, and group (DCD/control) as the between-subjects variable. The goodness of fit for the linear relation between movement duration and ID was significantly higher for the control group, $F(1,52)=4.47, p=.039, \eta^{2}=.079$. The goodness of fit was significantly higher for executed movements than for the imagined movements, $F(2,104)=161.30$, $p<.001, \eta^{2}=.756$. Furthermore, the goodness of fit increased with time, $F(2$, $104)=8.103, p=.001, \eta^{2}=.135$, and this did not differ between groups, $F(2$, $104)=0.898, p=.410, \eta^{2}=.017$. No other interactions were significant at an alpha level of .05. In Figure 4, the mean goodness of fit for each group at all three measurement occasions is displayed.

The slope of the linear relation between movement duration and ID was examined with a repeated-measures ANOVA with time (T0, T1, T2) as the within-subjects variable, 

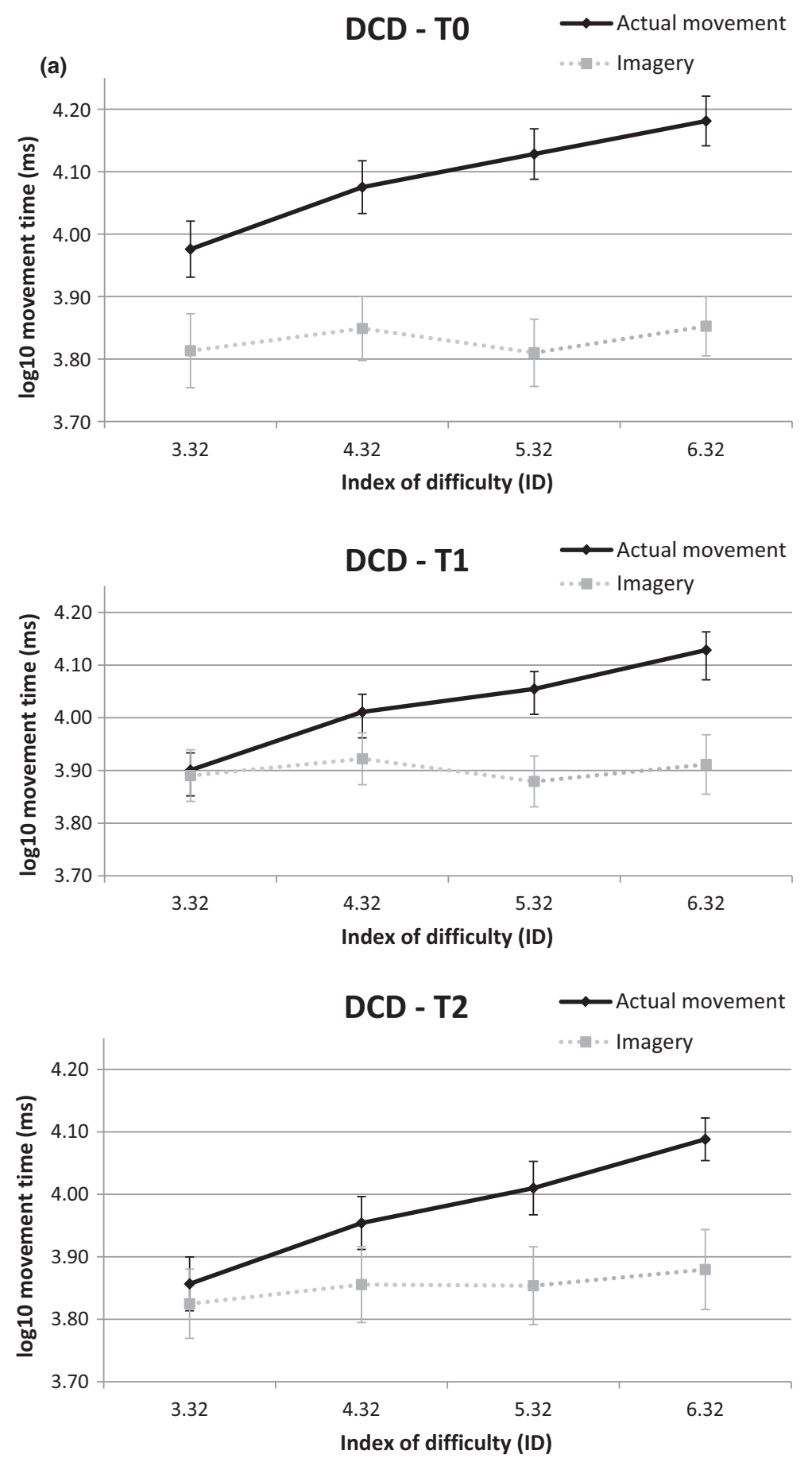

Figure 3. (A) Log 10 Movement time per index of difficulty (ID) for the developmental coordination disorder (DCD) group. Solid line (black) refers to executed movements, and dashed line (grey) refers to imagined movements. (B). Log 10 Movement time per D) for the control group. Solid line (black) refers to executed movements, and dashed line (grey) refers to imagined movements. 
196 Imke L. J. Adams et al.
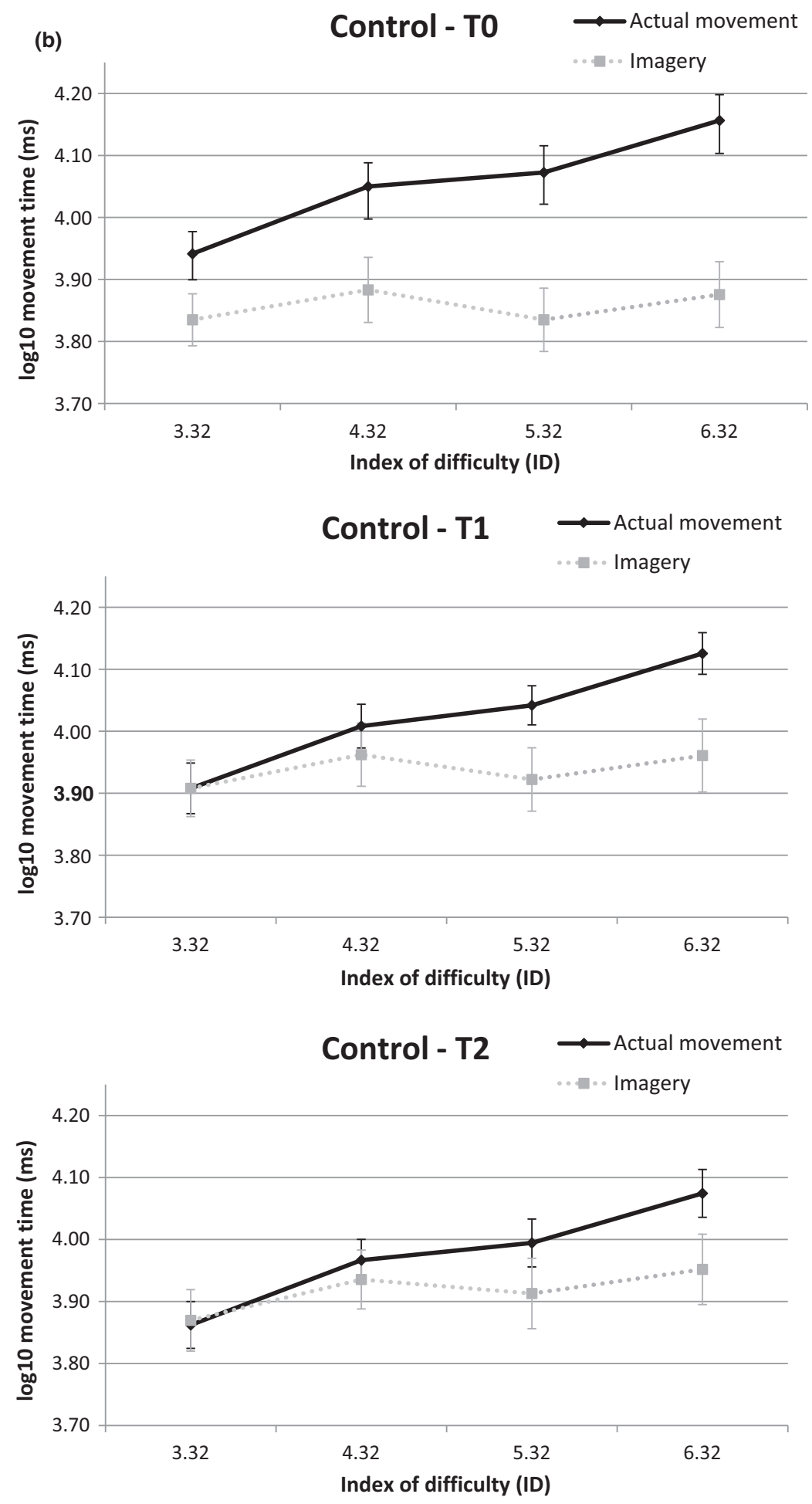

Figure 3. Continued 
Table 2. Results on linear fit for the developmental coordination disorder (DCD) and control group at all three measurements (T0, TI, T2)

\begin{tabular}{|c|c|c|c|c|c|c|}
\hline & \multicolumn{2}{|c|}{ T0 } & \multicolumn{2}{|c|}{ TI } & \multicolumn{2}{|c|}{$\mathrm{T} 2$} \\
\hline & DCD & Control & $\mathrm{DCD}$ & Control & DCD & Control \\
\hline \multicolumn{7}{|c|}{ Linear fit - execution } \\
\hline$R^{2}>0$ & $\begin{array}{l}t(26)=13.90 \\
p<.001\end{array}$ & $\begin{array}{l}t(26)=27.23 \\
p<.001\end{array}$ & $\begin{array}{l}t(26)=22.25 \\
p<.001\end{array}$ & $\begin{array}{l}t(26)=36.03 \\
p<.001\end{array}$ & $\begin{array}{l}t(26)=29.83, \\
p<.001\end{array}$ & $\begin{array}{l}t(26)=27.75, \\
p<.001\end{array}$ \\
\hline \multicolumn{7}{|c|}{ Linear fit - imagery } \\
\hline$R^{2}>0$ & $\begin{array}{l}t(26)=5.77 \\
p<.001\end{array}$ & $\begin{array}{l}t(26)=5.97 \\
p<.001\end{array}$ & $\begin{array}{l}t(26)=6.44 \\
p<.001\end{array}$ & $\begin{array}{l}t(26)=7.92 \\
p<.001\end{array}$ & $\begin{array}{l}t(26)=8.50 \\
p<.001\end{array}$ & $\begin{array}{l}t(26)=10.28, \\
p<.001\end{array}$ \\
\hline
\end{tabular}
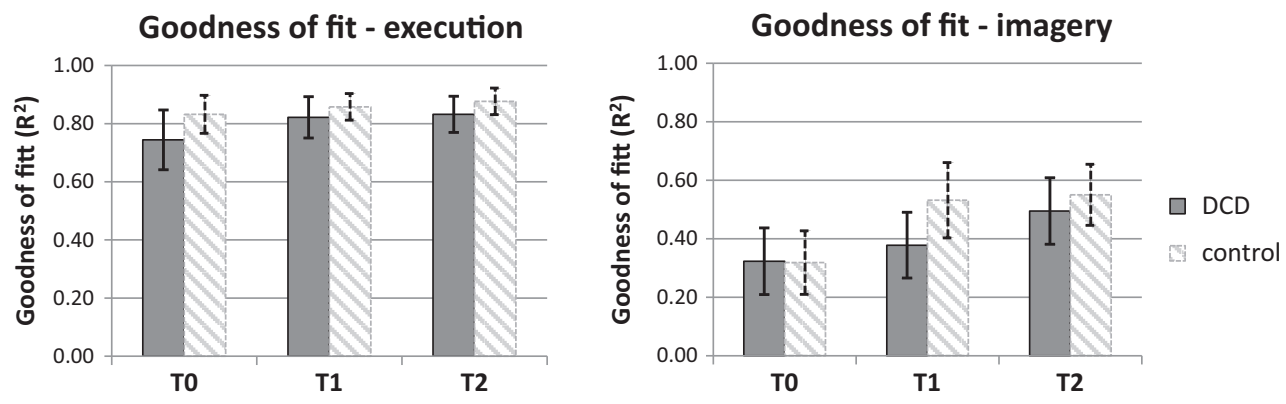

Figure 4. Goodness of fit for the linear relation between movement duration and index of difficulty for the execution and imagery performance for the developmental coordination disorder (DCD) and control group. Error bars represent $95 \% \mathrm{Cl}$.
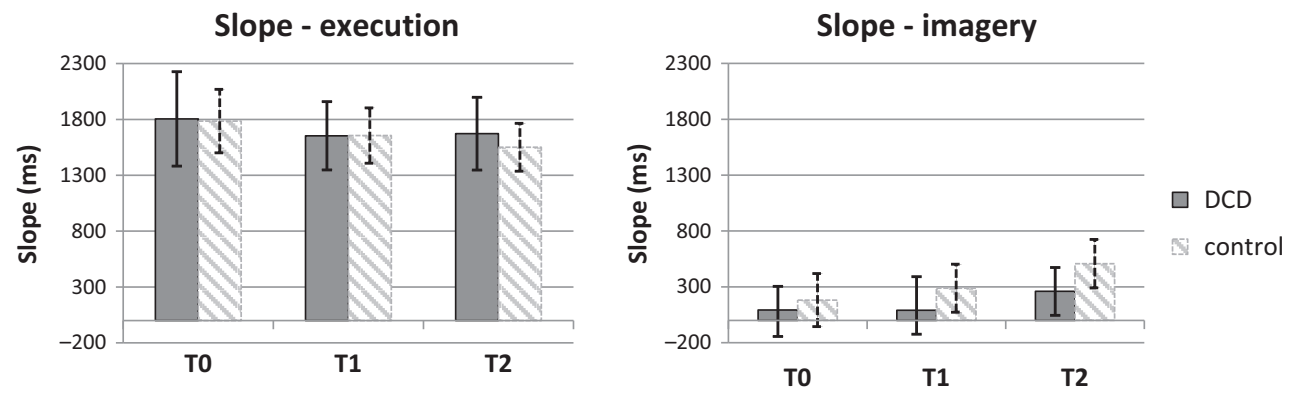

Figure 5. Slopes for the linear relation between movement duration and index of difficulty for the execution and imagery performance for the developmental coordination disorder (DCD) and control group. Error bars represent $95 \% \mathrm{Cl}$.

and group (DCD/control) as the between-subjects variable. The slope of the linear relation between movement duration and ID did not differ between groups $(p=.870)$, see also Figure 5 . The slope did not change over time, $F(2,104)=2.506, p=.087, \eta^{2}=.046$, but the slope of executed movements was significantly higher than the slope of imagined movements, $F(1,52)=219.31, p<.001, \eta^{2}=.81$. 


\section{Discussion}

In the present longitudinal study, we examined the development of explicit motor imagery ability in children with DCD via the use of the C-VRFT imagery task. Explicit motor imagery in these children was assessed in three subsequent measurements interspersed by one year. Our main research question was how the often reported compromised motor imagery ability in these children develops over time. In accordance with a developmental delay hypothesis (Hyde \& Wilson, 2013), it was expected that the DCD group would have a lower correlation between executed and imagined movements at the first measurement (T0), compared to controls, but that both groups would show a similar increase in correlation between executed and imagined movements over time. Results of the current study showed that children with DCD indeed had a significantly lower correlation between executed and imagined movements. In addition, children with DCD showed a lower linear fit compared to their typically developing peers indicating that the children with DCD obeyed to Fitts' law to a lesser extent than controls. Decreased linear fit in the DCD group indicates that these children had difficulty with performing the task, or did not follow the task instructions as closely as their peers. Importantly, the increase in the correlation and linear fit during subsequent measurements was comparable for the DCD and control group, in favour of the developmental delay hypothesis. Annaz et al. (2008) and Thomas et al.(2009) provided in-depth descriptions of developmental trajectories. Results of the present study are indicative of a delayed onset, as this trajectory is described as lower performance at T0, but a normal rate (not statistically different from the normal trajectory) of development. These results are in accordance with the recent study on implicit motor imagery ability in DCD (Adams et al., 2017a). To find out whether children with DCD eventually catch up with their typically developing peers, a longitudinal study with a longer time span is needed. For action planning, it has been shown that children with DCD aged 6-11 years do catch up with their typically developing peers during the 2-year follow-up (Adams et al., 2017a). In what follows, the results are discussed in comparison with earlier studies that used mental chronometry paradigms with varying complexity in children with DCD. Subsequently, the implications of the observed delayed motor imagery ability development in DCD for clinical rehabilitation are discussed.

The correlations between executed and imagined movement times reported in the present study are similar to that reported by Williams et al. (2013). They used the paperand-pencil VGPT to study explicit motor imagery ability in children aged 7-12 years. In contrast, the correlations found in the present study are lower than those presented in the study of Ferguson et al. (2015) (DCD $r_{\mathrm{s}}=.62$, control $r_{\mathrm{s}}=.82$ ). These differences could be due to differences in the C-VRFT that was used. In the present study, we varied both target width and amplitude, resulting in IDs of 3.32-6.32. In the study of Ferguson et al. (2015), only target width was varied and not amplitude. Five different target widths were used resulting in IDs of 2.9-6.9. The use of five different IDs, with a greater dispersion of IDs, might have led to the higher correlation in the study of Ferguson et al. (2015) than in the present study. This explanation is supported when comparing the results of the typically developing children in the present study to that reported by Spruijt, van der Kamp, and Steenbergen (2015). Like in our study, they used a C-VRFT in which both target width and distance were varied between trials, Pearson correlations of $0.16-0.41$ for children aged 6-8 years were reported. The results of the present study, in comparison with earlier studies, again testify the task specificity of the impaired predictive control of 
children with DCD. Subtle differences in task complexity between studies can affect the pattern of deficits observed (Adams, Ferguson, et al., 2016).

The present study extends previous studies by examining the development of explicit motor imagery ability. In contrast to cross-sectional studies that can only speculate on within-subject development, the current longitudinal data show a consistent difference over time in explicit motor imagery performance between children with DCD and agematched controls in favour of a delayed onset of motor imagery ability (Annaz et al., 2008; Hyde \& Wilson, 2013; Ruddock et al., 2015). From a clinical perspective, a delayed onset in DCD suggests that children have the potential to improve their explicit motor imagery ability. This strengthens the use of motor imagery training, for improvement of motor skills in children with DCD.

Current motor imagery interventions contain a mixture of implicit motor imagery (using action observation by means of videos) and explicit motor imagery (using verbal instructions for the imagery of movements) (Wilson et al., 2002, 2016). We hypothesize that to be able to make motor imagery interventions more tailor-made the decision whether implicit or explicit motor imagery exercises are used should be dependent on child characteristics such as age and motor imagery ability. Future studies should include a larger DCD group to be able to stratify results according to different age groups. This will give more detailed information from what age explicit motor imagery instructions can be used for children with DCD. Together, this will help to improve current motor imagery trainings and make such interventions more tailor-made.

Current longitudinal data on implicit and explicit MI ability (Adams et al., 2017a) uncovered a delayed onset in the development of this ability. Although clear group differences exist at each time point, the rate of improvement across time is similar between groups. A limitation in these studies is the broad age range that was included. An important question to ask is how the reported change over time is related to change over age. In the current study, we closely matched children in the DCD and control group on age to reduce between group variability. To distinguish development of MI ability across age, a study set-up with stratification in age groups is needed.

Another limitation is that we cannot rule out the possibility that cognitive (e.g., IQ, inhibitory control) or neuro-motor (e.g., general reaching efficiency) factors other than motor imagery ability influence performance on the C-VRFT (Choudhury, Charman, Bird, \& Blakemore, 2007; Gabbard, Lee, \& Cacola, 2013; Malouin, Belleville, Richards, Desrosiers, \& Doyon, 2004). We have excluded children with an ADHD diagnosis before analysis, preventing the confounding effect that severe attentional problems might have on motor imagery performance. Also, the C-VRFT was chosen instead of the VGPT because the C-VRFT has lower cognitive demands. When performing the VGPT, the participant must keep count of the number of completed movements, thus enlisting aspects of working memory (Ferguson et al., 2015). We therefore hypothesized that working memory problems would influence the performance of the C-VRFT less than in the VGPT. Future studies should have a closer look at which cognitive processes are related to the ability to use motor imagery.

To conclude, the present study showed that children with DCD are able to perform an explicit motor imagery task and that this ability improved over time; movement time increased with increasing ID and the correlation between executed and imagined movements increased during subsequent measurement occasions. However, the correlation between executed and imagined movements was significantly lower in the DCD group than in the control group, and although children with DCD improved their performance, they did not catch up with their typically developing peers. These results 
are in favour of a delayed onset of explicit motor imagery development in children with DCD. Longitudinal studies including a larger time frame than 2 years are needed to find out whether children with DCD eventually catch up with their typically developing peers. Inclusion of adolescents with DCD in such a longitudinal study might also clarify this issue. Together, these results provide an important avenue for training and rehabilitation of motor skills in children with DCD as improvement of explicit motor imagery ability is possible in DCD. Based on the current results, it seems likely that explicit motor imagery instructions can be used to improve motor imagery skills in children with DCD.

\section{Acknowledgements}

We gratefully acknowledge the participating children and their parents. We thank cooperating physical therapists, parent organization Balans, and the primary schools for their cooperation.

\section{Declaration of interest}

The authors report no declarations of interest.

\section{Funding}

This study was funded by the Graduate School of the Behavioural Science Institute Nijmegen, Radboud University, Nijmegen.

\section{References}

Adams, I. L., Ferguson, G. D., Lust, J. M., Steenbergen, B., \& Smits-Engelsman, B. C. (2016). Action planning and position sense in children with developmental coordination disorder. Human Movement Science, 46, 196-208. https://doi.org/10.1016/j.humov.2016.01.006

Adams, I. L., Lust, J. M., Wilson, P. H., \& Steenbergen, B. (2014). Compromised motor control in children with DCD: A deficit in the internal model? A systematic review. Neuroscience and Biobehavioral Reviews, 47C, 225-244. https://doi.org/10.1016/j.neubiorev.2014.08.011

Adams, I. L. J., Lust, J. M., Wilson, P. H., \& Steenbergen, B. (2017a). Development of motor imagery and action planning in children with developmental coordination disorder - A longitudinal approach. Human Movement Science, 55, 296-306. https://doi.org/10.1016/j.humov.2017. 08.021

Adams, I. L. J., Lust, J. M., Wilson, P. H., \& Steenbergen, B. (2017b). Testing predictive control of movement in children with developmental coordination disorder using converging operations. British Journal of Psychology, 108(1), 73-79. https://doi.org/10.1111/bjop.12183

Adams, I. L., Steenbergen, B., Lust, J. M., \& Smits-Engelsman, B. C. (2016). Motor imagery training for children with developmental coordination disorder - Study protocol for a randomized controlled trial. BMC Neurology, 16(1), 5. https://doi.org/10.1186/s12883-016-0530-6

American Psychiatric Association (2013). Diagnostic and statistical manual of mental disorders (5th ed.). Washington, DC: Author.

Annaz, D., Karmiloff-Smith, A., \& Thomas, M. S. C. (2008). The importance of tracing developmental trajectories for clinical child neuropsychology. In J. Reed \& J. Warner Rogers (Eds.), Child neuropsychology concepts, theory practice (pp. 7-18). Oxford, UK: Wiley-Blackwell.

Caeyenberghs, K., Tsoupas, J., Wilson, P. H., \& Smits-Engelsman, B. C. M. (2009). Motor imagery development in primary school children. Developmental Neuropsychology, 34(1), 103-121. https://doi.org/10.1080/87565640802499183 
Caeyenberghs, K., Wilson, P. H., van Roon, D., Swinnen, S. P., \& Smits-Engelsman, B. C. (2009). Increasing convergence between imagined and executed movement across development: Evidence for the emergence of movement representations. Developmental Science, 12, 474483. https://doi.org/10.1111/j.1467-7687.2008.00803.x

Choudhury, S., Charman, T., Bird, V., \& Blakemore, S. J. (2007). Adolescent development of motor imagery in a visually guided pointing task. Consciousness and Cognition, 16, 886-896. https:// doi.org/10.1016/j.concog.2006.11.001

Decety, J., \& Grezes, J. (1999). Neural mechanisms subserving the perception of human actions. Trends in Cognitive Science, 3(5), 172-178. https://doi.org/S1364-6613(99)01312-1 [pii]

Decety, J., Jeannerod, M., \& Prablanc, C. (1989). The timing of mentally represented actions. Behavioural Brain Research, 34(1-2), 35-42.

Deconinck, F. J., Spitaels, L., Fias, W., \& Lenoir, M. (2009). Is developmental coordination disorder a motor imagery deficit? Journal of Clinical Experimental Neuropsychology, 31, 720-730. https://doi.org/10.1080/13803390802484805

Ferguson, G. D., Wilson, P. H., \& Smits-Engelsman, B. C. (2015). The influence of task paradigm on motor imagery ability in children with developmental coordination disorder. Human Movement Science, 44, 81-90. https://doi.org/10.1016/j.humov.2015.08.016

Fitts, P. M. (1954). The information capacity of the human motor system in controlling the amplitude of movement. Journal of Experimental Psychology, 47, 381-391. https://doi.org/10.1037/ H0055392

Fong, S. S., Chung, J. W., Cheng, Y. T., Yam, T. T., Chiu, H. C., Fong, D. Y., .. Ng, S. S. (2016). Attention during functional tasks is associated with motor performance in children with developmental coordination disorder: A cross-sectional study. Medicine (Baltimore), 95, e4935. https://doi.org/10.1097/MD.0000000000004935

Fuelscher, I., Williams, J., Enticott, P. G., \& Hyde, C. (2015). Reduced motor imagery efficiency is associated with online control difficulties in children with probable developmental coordination disorder. Research in Developmental Disabilities, 45-46, 239-252. https://doi. org/10.1016/j.ridd.2015.07.027

Fuelscher, I., Williams, J., Wilmut, K., Enticott, P. G., \& Hyde, C. (2016). Modeling the maturation of grip selection planning and action representation: Insights from typical and atypical motor development. Frontiers in Psychology, 7, 108. https://doi.org/10.3389/fpsyg. 2016.00108

Gabbard, C. (2009). Studying action representation in children via motor imagery. Brain and Cognition, 71, 234-239. https://doi.org/10.1016/j.bandc.2009.08.011

Gabbard, C., Lee, J., \& Cacola, P. (2013). Role of working memory in transformation of visual and motor representations for use in mental simulation. Cognitive Neuroscience, 4, 210-216. https://doi.org/10.1080/17588928.2013.820180

Goulardins, J. B., Rigoli, D., Licari, M., Piek, J. P., Hasue, R. H., Oosterlaan, J., \& Oliveira, J. A. (2015). Attention deficit hyperactivity disorder and developmental coordination disorder: Two separate disorders or do they share a common etiology. Behavioural Brain Research, 292, 484-492. https://doi.org/10.1016/j.bbr.2015.07.009

Henderson, S. E., Sugden, D. A., \& Barnett, A. L. (2007). Movement Assessment Battery for Children (2nd ed., examiner's manual). London, UK: Harcourt Assessment.

Hétu, S., Grégoire, M., Saimpont, A., Coll, M., Eugène, F., Michon, P., \& Jackson, P. L. (2013). The neural network of motor imagery: An ALE meta-analysis. Neuroscience and Biobehavioral Reviews, 37, 930-949. https://doi.org/10.1016/j.neubiorev.2013.03.017

Hyde, C., Fuelscher, I., Buckthought, K., Enticott, P. G., Gitay, M. A., \& Williams, J. (2014). Motor imagery is less efficient in adults with probable developmental coordination disorder: Evidence from the hand rotation task. Research in Developmental Disabilities, 35, 3062-3070. https:// doi.org/10.1016/j.ridd.2014.07.042

Hyde, C. E., \& Wilson, P. H. (2013). Impaired online control in children with developmental coordination disorder reflects developmental immaturity. Developmental Neuropsychology, 38(2), 81-97. https://doi.org/10.1080/87565641.2012.718820 
Lewis, M., Vance, A., Maruff, P., Wilson, P., \& Cairney, S. (2008). Differences in motor imagery between children with developmental coordination disorder with and without the combined type of ADHD. Developmental Medicine and Child Neurology, 50, 608-612. https://doi.org/ 10.1111/j.1469-8749.2008.03030.x

Malouin, F., Belleville, S., Richards, C. L., Desrosiers, J., \& Doyon, J. (2004). Working memory and mental practice outcomes after stroke. Archives of Physical Medicine and Rehabilitation, 85, $177-183$.

Maruff, P., Wilson, P., Trebilcock, M., \& Currie, J. (1999). Abnormalities of imaged motor sequences in children with developmental coordination disorder. Neuropsychologia, 37, 1317-1324. https://doi.org/S0028393299000160

Noten, M., Wilson, P., Ruddock, S., \& Steenbergen, B. (2014). Mild impairments of motor imagery skills in children with DCD. Research in Developmental Disabilities, 35, 1152-1159. https:// doi.org/10.1016/j.ridd.2014.01.026

Papaxanthis, C., Pozzo, T., Skoura, X., \& Schieppati, M. (2002). Does order and timing in performance of imagined and actual movements affect the motor imagery process? The duration of walking and writing task. Behavioural Brain Research, 134(1-2), 209-215. https://doi.org/ 10.1016/S0166-4328(02)00030-X

Prunty, M. M., Barnett, A. L., Wilmut, K., \& Plumb, M. S. (2013). Handwriting speed in children with developmental coordination disorder: Are they really slower? Research in Developmental Disabilities, 34, 2927-2936. https://doi.org/10.1016/j.ridd.2013.06.005

Rosenblum, S., \& Livneh-Zirinski, M. (2008). Handwriting process and product characteristics of children diagnosed with developmental coordination disorder. Human Movement Science, 27, 200-214. https://doi.org/10.1016/j.humov.2008.02.011

Ruddock, S., Piek, J., Sugden, D., Morris, S., Hyde, C., Caeyenberghs, K., \& Wilson, P. (2015). Coupling online control and inhibitory systems in children with developmental coordination disorder: Goal-directed reaching. Research in Developmental Disabilities, 36, 244-255. https://doi.org/10.1016/j.ridd.2014.10.013

Schoemaker, M. M., Reinders-Messelink, A. J., \& de Kloet, A. J. (2008). Coördinatievragenlijst voor Ouders (CVO) [Coordination Questionnaire for Parents]-Translation of DCD Questionnaire by Wilson (2007). Groningen, the Netherlands: University of Groningen

Scholte, E. M., \& van der Ploeg, J. D. (2004). ADHD-vragenlijst (AVL) [ADHD questionnaire]. Amsterdam, the Netherlands: Nippo-Nederlands Instituut voor Pedagogisch en Psychologisch Onderzoek.

Sirigu, A., Duhamel, J. R., Cohen, L., Pillon, B., Dubois, B., \& Agid, Y. (1996). The mental representation of hand movements after parietal cortex damage. Science, 273, 1564-1568.

Smits-Engelsman, B. C. M. (2010). Handleiding movement ABC-2-NL [Users manual movement $A B C-2]$. Amsterdam, the Netherlands: Pearson.

Smits-Engelsman, B. C., \& Wilson, P. H. (2013). Age-related changes in motor imagery from early childhood to adulthood: Probing the internal representation of speed-accuracy tradeoffs. Human Movement Science, 32, 1151-1162. https://doi.org/10.1016/j.humov.2012.06. 006

Spruijt, S., Jouen, J., Molina, M., Kudlinski, C., Guilbert, J., \& Steenbergen, B. (2013). Assessment of motor imagery in cerebral palsy via mental chronometry: The case of walking. Research in Developmental Disabilities, 34, 4154-4160. https://doi.org/10.1016/j.ridd.2013.08.044

Spruijt, S., van der Kamp, J., \& Steenbergen, B. (2015). The ability of 6-to 8-year-old children to use motor imagery in a goal-directed pointing task. Journal of Experimental Child Psychology, 139, 221-233. https://doi.org/10.1016/j.jecp.2015.06.008

Steenbergen, B., van der Kamp, J., Verneau, M., Jongbloed-Pereboom, M., \& Masters, R. S. (2010). Implicit and explicit learning: Applications from basic research to sports for individuals with impaired movement dynamics. Disability and Rehabilitation, 32, 1509-1516. https://doi.org/ $10.3109 / 09638288.2010 .497035$ 
ter Horst, A. C., van Lier, R., \& Steenbergen, B. (2010). Mental rotation task of hands: Differential influence number of rotational axes. Experimental Brain Research, 203, 347-354. https://doi. org/10.1007/s00221-010-2235-1

Thomas, M. S. C., Annaz, D., Ansari, D., Scerif, G., Jarrold, C., \& Karmiloff-Smith, A. (2009). Using developmental trajectories to understand developmental disorders. Journal of Speech, Language, and Hearing Research, 52, 336-358. https://doi.org/10.1044/1092-4388(2009/ 07-0144)

Williams, J., Omizzolo, C., Galea, M. P., \& Vance, A. (2013). Motor imagery skills of children with attention deficit hyperactivity disorder and developmental coordination disorder. Human Movement Science, 32(1), 121-135. https://doi.org/10.1016/j.humov.2012.08.003

Williams, J., Thomas, P. R., Maruff, P., \& Wilson, P. H. (2008). The link between motor impairment level and motor imagery ability in children with developmental coordination disorder. Human Movement Science, 27, 270-285. https://doi.org/10.1016/j.humov.2008.02.008

Wilson, P. H., Adams, I. L., Caeyenberghs, K., Thomas, P., Smits-Engelsman, B., \& Steenbergen, B. (2016). Motor imagery training enhances motor skill in children with DCD: A replication study. Research in Developmental Disabilities, 57, 54-62. https://doi.org/10.1016/j.ridd.2016.06. 014

Wilson, P. H., \& Butson, M. (2007). Deficits underlying DCD (Chapter 4). In R. H. Geuze (Ed.), Developmental coordination disorder: A review of current approaches (pp. 115-119). Marseille, France: Solal Editeurs.

Wilson, P. H., Maruff, P., Ives, S., \& Currie, J. (2001). Abnormalities of motor and praxis imagery in children with DCD. Human Movement Science, 20(1-2), 135-159.

Wilson, P. H., Ruddock, S., Smits-Engelsman, B., Polatajko, H., \& Blank, R. (2013). Understanding performance deficits in developmental coordination disorder: A meta-analysis of recent research. Developmental Medicine and Child Neurology, 55, 217-228. https://doi.org/10. 1111/j.1469-8749.2012.04436.x

Wilson, P. H., Smits Engelsman, B. C., Caeyenberghs, K., Steenbergen, B., Sugden, D., Clark, J. E., . . Blank, R. (2017). Cognitive and neuroimaging findings in developmental coordination disorder: New insights from a systematic review of recent research. Developmental Medicine $\&$ Child Neurology, 59 (11), 1117-1129. https://doi.org/10.1111/dmcn.13530

Wilson, P. H., Thomas, P. R., \& Maruff, P. (2002). Motor imagery training ameliorates motor clumsiness in children. Journal of Child Neurology, 17, 491-498.

Wolpert, D. M. (1997). Computational approaches to motor control. Trends in Cognitive Science, 1 , 209-216. https://doi.org/10.1016/S1364-6613(97)01070-X

Received 3 March 20 I7; revised version received 19 September 2017 